\title{
Psychosocial Stress in Nurses With Shift Work Schedule Is Associated With Functional Gastrointestinal Disorders
}

\author{
Seong-Joon Koh, Mingoo Kim, Da Yeon Oh, Byeong Gwan Kim, Kook Lae Lee and Ji Won Kim* \\ Department of Internal Medicine, Seoul National University Boramae Hospital, Seoul National University College of Medicine, Seoul, Korea
}

\section{Background/Aims}

The aim of this study was to investigate the role of psychosocial problems and their associations with rotating shift work in the development of functional gastrointestinal disorders.

\section{Methods}

In this cross-sectional observation study, survey was administered to nurses and nurse assistants in a referral hospital. In addition to demographic questions, subjects were asked to complete the Rome III Questionnaire, Pittsburgh Sleep Quality Index and Rome III Psychosocial Alarm Questionnaire.

\section{Results}

Responses from 301 subjects were assessed. The overall prevalence of irritable bowel syndrome (IBS) and functional dyspepsia (FD) were $15.0 \%$ and $19.6 \%$, respectively. Psychosocial alarms were prevalent in the nursing personnel $(74.8 \%$ with alarm presence and $23.3 \%$ with serious condition) and were more frequent among rotating shift workers $(84.7 \%$ vs. $74.5 \%$ for alarm presence and $28.1 \%$ vs. $13.3 \%$ for serious condition). The prevalence of both IBS and FD significantly increased with psychosocial risk. An independent risk factor for IBS was serious psychosocial alarm (adjusted odds ratio [aOR], 10.75; 95\% confidence interval $(C l), 1.30-88.99 ; P=0.028)$. Serious psychosocial alarm was an independent risk factor for $\mathrm{FD}(\mathrm{aOR}, 7.84 ; 95 \% \mathrm{Cl}$, $1.98-31.02 ; P=0.003)$. Marriage $(a O R \quad 0.30 ; 95 \% \mathrm{Cl}, 0.09-0.93 ; P=0.037)$ was associated with the decreased risk of FD.

\section{Conclusions}

The high prevalence of psychosocial stress among nurses who work rotating shifts is associated with the development of functional gastrointestinal disorders.

\section{(J Neurogastroenterol Motil 2014;20:516-522)}

\section{Key Words}

Dyspepsia; Irritable bowel syndrome; Marriage; Sleep

Received: March 25, 2014 Revised: May 15, 2014 Accepted: May 17, 2014

(c) This is an Open Access article distributed under the terms of the Creative Commons Attribution Non-Commercial License (http://creativecommons. org/licenses/by-nc/3.0) which permits unrestricted non-commercial use, distribution, and reproduction in any medium, provided the original work is properly cited.

*Correspondence: Ji Won Kim, MD, PhD

Department of Internal Medicine, Seoul National University Boramae Hospital, Seoul National University College of Medicine, 20 , Boramae-ro 5-gil, Dongjak-gu, Seoul 156-707, Korea

Tel: +82-2-870-2221, Fax: +82-2-870-3866, E-mail: kjwjor@snu.ac.kr

Seong-Joon Koh and Mingoo Kim contributed equally to this work.

Financial support: None.

Conflicts of interest: None.

Author contributions: Ji Won Kim and Seong-Joon Koh, planned the study; Mingoo Kim, collected the data and analyzed the results; Mingoo Kim, Seong-Joon Koh and Ji Won Kim, wrote the paper; Byeong Gwan Kim, Da Yeon Oh and Kook Lae Lee, interpreted the data and reviewed the paper.

ORCID: Ji Won Kim, http://orcid.org/0000-0002-1214-5544. 


\section{Introduction}

Functional gastrointestinal disorders (FGIDs), which encompass both irritable bowel syndrome (IBS) and functional dyspepsia (FD), are defined as a group of gastrointestinal disorders that are characterized by upper and lower symptoms with no definite organic or biochemical abnormalities that can explain symptoms. ${ }^{1,2}$ Although risk factors for functional gastrointestinal disorders (FGIDs) have been much studied, conflicting results have been reported; therefore, the pathogenesis remains unclear. ${ }^{3}$

Psychosocial factors have been proposed as a cause of IBS. Higher rates of IBS have been observed in psychiatric patients. ${ }^{4}$ Psychiatric symptoms are more common among subjects who have IBS than those who do not. ${ }^{5}$ In a study of enrolled medical students and interns, morbid anxiety was one of the independent predictors of IBS. ${ }^{6}$ Although, the basic role of psychosocial factors in the development of FGIDs remains obscure, these studies have provided a basis for understanding the role of psychosocial factors in developing FGIDs.

It has been suggested that shift work, poor sleep quality, and the additional role of circadian rhythm disruption are all risk factors for FGIDs in nursing professionals. ${ }^{7,8}$ Previous publications focused on the link between shift work and gastrointestinal symptoms. In a previous study, participations in rotating shift work showed a significantly higher risk of IBS. ${ }^{8}$ The association between rotating shift work and IBS was also observed in a subsequent study performed in Korea. ${ }^{7}$ Both studies found the significant association between rotating shift work and IBS after controlling for sleep quality in multivariate regression analysis, which suggested that circadian rhythm disruption might be an additional contributing factor for the pathogenesis of IBS. Although these prior studies provided evidence for a relationship between rotating shift work and the prevalence of FGIDs, they did not consider psychosocial factors in their analysis. In fact, these studies excluded subjects who used antidepressants or sedatives, and they disregarded psychosocial factors in subsequent analyses. This may cause bias because subclinical psychosocial symptoms not requiring psychiatric medications could influence the development of IBS or FD. Therefore, there is a great need for conducting studies that evaluate the role of psychosocial aspects and their association with rotating shift work in the development of FGIDs. A more thorough examination of these factors would help to elucidate the role of psychosocial problems between rotating shift work and FGIDs.
In the present study, we sought to explain the link between shift work and FGIDs in nursing professionals. We included psychosocial distress into our analysis in addition to demographic variables, GI symptoms, shift work schedules and sleep quality. We compared the prevalence of psychosocial problems between nursing professionals working different shift schedules and evaluated the association of psychosocial problems and the presence of IBS and FD.

\section{Materials and Methods}

\section{Study Design and Setting}

This was a cross-sectional observational study of hospital nurses and nurse assistants employed at Seoul National University Boramae Medical Center, a referral hospital in a major metropolitan area in Korea. This study was done between July 2012 and February 2013.

\section{Study Subjects}

All nurses and nurse assistants working at Boramae Medical Center were recruited for this study on a voluntary basis. The subjects completed self-reporting paper-based questionnaires that assessed their bowel symptoms, psychosocial status, sleep quality and demographic characteristics. The exclusion criteria for the subjects included chronic illnesses that would be expected to contribute to gastrointestinal symptoms, such as cancer, inflammatory bowel disease or hyperthyroidism and previous abdominal surgery. In addition, subjects who were currently pregnant or breast feeding or were on a prescribed course of gastrointestinal or psychological medication were excluded from this study. Incomplete responses were also excluded from the subsequent analysis. The Institutional Review Boards of Boramae Hospital approved the study (IRB No. 06-2012-18), and each respondent signed a written informed consent form.

\section{Questionnaires and Outcome Measures}

Four sets of questionnaires, comprising 61 questions, were used. For the diagnosis of IBS and FD based on the Rome III criteria, a Korean version of Bowel Disease Questionnaire was used. ${ }^{9}$ To measure sleep quality, we used the Pittsburgh Sleep Quality Index (PSQI).$^{10}$ For the assessment of psychosocial status, we used the Rome III Psychosocial Alarm Questionnaire, which helped to identify the psychosocial problems commonly faced by patients with FGIDs. ${ }^{11}$ The Rome III Psychosocial 
Alarm Questionnaire includes the following 7 questions regarding anxiety, depression, suicidal ideas and other psychosocial distress factors designed to identify subjects with psychosocial problems: (1) In the last week, have you felt tense, or "wound up"?; (2) In the last week, have you felt downhearted and low?; (3) Have you recently felt so low that you felt like hurting or killing yourself?; (4) During the last 4 weeks, how much bodily pain have you had?; (5) During the last 4 weeks, how much did pain interfere with your normal activities?; (6) When I have pain, I say to myself "it is terrible, and I feel it will never get better"; and (7) It is quite common for people to have been emotionally, physically or sexually victimized at some time in their lives, and this can affect how people manage their medical conditions. Has this ever happened to you? According to previous studies, psychosocial alarm was defined as being non-serious if a respondent answered "yes" to any of the questions not covering a serious situation, and it was defined as serious if any positive response was given to questions regarding a serious situation. ${ }^{12}$ In the self-administered questionnaire, data regarding age, sex, height, weight, education, marriage, chronic medical conditions, medical history and medication used, work duration, and shift work status were included.

\section{Statistical Methods}

All analyses were performed with SPSS version 20 (IBM, New York, USA). Continuous variables were evaluated by an independent unpaired $t$ test. Categorical data were evaluated by a Chi-square or Fisher's exact test. Logistic regression analysis was used to assess the possible risk factors for IBS and FD. A $P$-value $<0.2$ in the univariate analysis was required for entry into a binary logistic regression analysis model. Additionally, the variables evaluated as risk factors for the development of IBS and FD were selected from previous reports and the authors' experience. A $P$-value threshold of 0.05 was used, unless otherwise noted.

\section{Results}

\section{Subject Characteristics}

Of 607 nurses and nurse assistants working in Boramae Hospital, 350 (57\%) volunteered to participate in this study. After excluding 25 people who response incompletely and 24 people who fit the exclusion criteria, a total of 301 subjects were included in the final analyses.

The baseline characteristics of the 301 nursing employees are shown in Table 1. Among the subjects, 88 had FGIDs (IBS or FD). Compared with the subjects without FGIDs, the FGID subjects were younger and had less work experience. More subjects without FGIDs were married. There were no significant differences in body mass index, gender, alcohol consumption or rotating shift work between the 2 groups. Nursing workers with FGIDs reported poorer sleep quality (represented by higher PSQI scores). Despite the higher scores, the proportions of poor sleepers (defined as PSQI scores $\geq 6$ ) were not significantly different between the 2 working groups.

Table 1. Baseline Characteristics of Study Subjects According to Functional Gastrointestinal Disorders

\begin{tabular}{lccc}
\hline & With FGIDs $(\mathrm{n}=88)$ & Without FGIDs $(\mathrm{n}=213)$ & $P$-value \\
\hline Age $(\mathrm{yr})$ & $28.12 \pm 4.88$ & $29.48 \pm 6.43$ & 0.050 \\
BMI $\left(\mathrm{kg} / \mathrm{m}^{2}\right)$ & $19.97 \pm 3.96$ & $20.19 \pm 3.61$ & 0.748 \\
Gender (Female) & $86 / 88(97.7)$ & $203 / 211(96.2)$ & 0.729 \\
Marriage (Married) & $18 / 88(20.5)$ & $72 / 211(33.6)$ & 0.026 \\
Education (4 year college graduate or higher) & $53 / 86(61.6)$ & $106 / 196(54.1)$ & 0.297 \\
Drinking ( $\geq$ 1 day/wk) & $48 / 88(54.5)$ & $112 / 207(54.1)$ & 1.000 \\
Work duration (mo) & $51.38 \pm 51.82$ & $68.97 \pm 77.37$ & 0.023 \\
Work experience ( $\geq 3$ yr) & $44 / 88(50.0)$ & $113 / 210(53.8)$ & 0.611 \\
Length of sleep (hr) & $8.14 \pm 1.97$ & $7.92 \pm 1.89$ & 0.433 \\
Sleep score (PSQI) & $10.45 \pm 2.94$ & $9.21 \pm 2.62$ & 0.001 \\
Poor sleeper (PSQI $\geq 6)$ & $71 / 75(94.7)$ & $174 / 188(92.6)$ & 0.787 \\
Rotating Shift work & $65 / 88(73.9)$ & $138 / 213(64.8)$ & 0.138 \\
\hline
\end{tabular}

FGIDs, functional gastrointestinal disorders; PSQI, Pittsburgh Sleep Quality Index.

Data presented as mean $\pm \mathrm{SD}$ or $\mathrm{n}[\%]$. 
Table 2. Prevalence of Functional Gastrointestinal Disorders Between Shift Work Types

\begin{tabular}{lcccc}
\hline & $\begin{array}{c}\text { All workers }(\mathrm{n}[\%]) \\
(\mathrm{N}=301)\end{array}$ & $\begin{array}{c}\text { Rotating shift workers (n [\%]) } \\
(\mathrm{n}=203)\end{array}$ & $\begin{array}{c}\text { Day shift workers (n [\%]) } \\
(\mathrm{n}=98)\end{array}$ & $P$-value \\
\hline IBS & $45(15.0)$ & $33(16.3)$ & $12(12.2)$ & 0.360 \\
IBS-C & $8(2.7)$ & $5(2.5)$ & $3(3.1)$ & 0.718 \\
IBS-D & $15(5.0)$ & $9(4.4)$ & $6(6.1)$ & 0.528 \\
IBS-M & $21(7.0)$ & $18(8.9)$ & $3(3.1)$ & 0.089 \\
IBS-U & $1(0.3)$ & $1(0.5)$ & $0(0.0)$ & 1.000 \\
FD & $59(19.6)$ & $45(22.2)$ & $14(14.3)$ & 0.107 \\
\hline
\end{tabular}

IBS, irritable bowel syndrome; IBS-C, constipation predominant IBS; IBS-D, diarrhea predominant IBS; IBS-M, mixed IBS; IBS-U, unsubtyped IBS; FD, functional dyspepsia.

Table 3. Prevalence of the Psychosocial Alarm Between Shift Work Types

\begin{tabular}{lcccc}
\hline & All workers (n [\%]) & Rotating shift workers (n [\%]) & Day shift workers (n [\%]) & (n -value \\
& $(\mathrm{N}=301)$ & $(\mathrm{n}=203)$ & $73(74.5)$ & 0.032 \\
Alarm present & $225(74.8)$ & $172(84.7)$ & $13(13.3)$ & 0.004 \\
Alarm serious & $70(23.3)$ & $57(28.1)$ & & 0 \\
\hline
\end{tabular}

Table 4. Prevalence of Functional Gastrointestinal Disorder According to the Psychosocial Alarm

\begin{tabular}{lcccr}
\hline & $\begin{array}{c}\text { Alarm absent }(\mathrm{n}[\%]) \\
(\mathrm{n}=56)\end{array}$ & $\begin{array}{c}\text { Alarm present }(\mathrm{n}[\%]) \\
(\mathrm{n}=175)\end{array}$ & $\begin{array}{c}\text { Alarm serious (n [\%]) } \\
(\mathrm{n}=70)\end{array}$ & $P$-value \\
\hline IBS & $2(3.6)$ & $43(17.6)$ & $18(25.7)$ & 0.001 \\
FD & $5(8.9)$ & $54(22.0)$ & $25(35.7)$ & $<0.001$ \\
\hline
\end{tabular}

IBS, irritable bowel syndrome; FD, functional dyspepsia.

\section{Prevalence of Irritable Bowel Syndrome and Functional Dyspepsia}

Overall, 45 workers were diagnosed as IBS sufferers, with a prevalence of $15 \%$ (Table 2). IBS was more common in the rotating shift group than in the day shift group, but there was no statistical significance $(16.3 \%$ vs. $12.2 \%, P=0.360)$. When IBS subtypes were considered, 21 (46.7\%) of the 45 IBS patients had mixed IBS, 15 (33.3\%) had diarrhea predominant IBS, 8 (17.8\%) had constipation predominant IBS and 1 (2.2\%) had unsubtyped IBS. No significant differences in prevalence were observed between the shift working groups for any of the IBS subtypes.

Fifty-nine workers fulfilled the Rome III criteria for FD with an overall prevalence of $19.6 \%$ (Table 2). FD was more common in rotating shift workers than day shift workers, but the difference was not statistically significant $(22.2 \%$ vs. $14.3 \%, P=0.107)$.

\section{Psychosocial Alarm}

As shown in Table 3, a total of 225 (74.8\%) of the respondents reported the presence of psychosocial alarm, and 70 (23.3\%) re- ported the serious alarm condition. The risk of psychosocial alarm was significantly greater among rotating shift workers than among day shift ones, for both the presence of alarm $(P=0.032)$ and for the seriousness of alarm $(P=0.004)$. In Table 4 , the prevalence of IBS and FD was shown to be stratified by psychosocial alarm. The prevalence of both IBS and FD significantly increased with psychosocial risk (IBS, $P=0.001$ FD, $P<0.001$ ).

\section{Risk Factors for Irritable Bowel Syndrome and Functional Dyspepsia}

Risk factors for the presence of IBS and FD are shown in Tables 5 and 6, respectively. Serious psychosocial alarm was associated with the increased risk of IBS in the univariate analysis. When adjusting for variables including rotating shift work and sleep quality, serious psychosocial alarm (adjusted odds ratio $[\mathrm{aOR}], 10.75$; $95 \%$ confidence interval (CI), 1.30-88.99; $P=$ 0.028 ) was the independent risk factor for IBS in the multivariate analysis. In FD, non-serious and serious psychosocial alarm were associated with an increased risk of FD. In multivariate analysis, 
Table 5. Risk Factors for Irritable Bowel Syndrome in Univariate and Multivariate Analyses

\begin{tabular}{|c|c|c|c|c|c|c|}
\hline & \multicolumn{3}{|c|}{ Univariate analysis } & \multicolumn{3}{|c|}{ Multivariate analysis } \\
\hline & OR & $95 \% \mathrm{CI}$ & $P$-value & OR & $95 \% \mathrm{CI}$ & $P$-value \\
\hline Rotating shift work & 1.39 & $0.68-2.82$ & 0.362 & 1.32 & $0.47-3.74$ & 0.596 \\
\hline Age (yr) & 0.98 & $0.99-1.04$ & 0.427 & 1.02 & $0.94-1.12$ & 0.603 \\
\hline $\mathrm{BMI}\left(\geq 18.5 \mathrm{~kg} / \mathrm{m}^{2}\right)$ & 0.72 & $0.32-1.61$ & 0.417 & 0.81 & $0.33-1.97$ & 0.644 \\
\hline Marriage (Married) & 0.73 & $0.35-1.52$ & 0.398 & 0.98 & $0.33-2.98$ & 0.981 \\
\hline Work experience ( $\geq 3 \mathrm{yr}$ ) & 0.84 & $0.44-1.58$ & 0.580 & & & \\
\hline Drinking ( $>1$ day/wk) & 0.96 & $0.51-1.81$ & 0.985 & & & \\
\hline College graduate & 1.97 & $0.98-3.96$ & 0.058 & 1.90 & $0.87-4.16$ & 0.107 \\
\hline Poor sleep quality (PSQI $\geq 6$ ) & 0.86 & $0.24-3.13$ & 0.820 & & & \\
\hline \multicolumn{7}{|l|}{ Psychosocial alarm } \\
\hline None & Reference & & & Reference & & \\
\hline Non-serious & 4.50 & $1.03-19.64$ & 0.045 & 7.43 & $0.97-57.21$ & 0.054 \\
\hline Serious & 9.35 & $2.07-42.29$ & 0.004 & 10.75 & $1.30-88.99$ & 0.028 \\
\hline
\end{tabular}

BMI, body mass index; PSQI, Pittsburgh Sleep Quality Index.

For the psychosocial alarm, the absence of psychosocial alarm is the reference group.

Table 6. Risk factors for Functional Dyspepsia in Univariate and Multivariate Analyses

\begin{tabular}{|c|c|c|c|c|c|c|}
\hline & \multicolumn{3}{|c|}{ Univariate analysis } & \multicolumn{3}{|c|}{ Multivariate analysis } \\
\hline & OR & $95 \% \mathrm{CI}$ & $P$-value & OR & $95 \% \mathrm{CI}$ & $P$-value \\
\hline Rotating shift work & 1.71 & $0.89-3.29$ & 0.109 & 0.71 & $0.29-1.70$ & 0.438 \\
\hline Age (yr) & 0.95 & $0.90-1.01$ & 0.081 & 1.03 & $0.95-1.11$ & 0.530 \\
\hline $\mathrm{BMI}\left(\geq 18.5 \mathrm{~kg} / \mathrm{m}^{2}\right)$ & 0.63 & $0.31-1.29$ & 0.211 & 1.66 & $0.61-4.51$ & 0.318 \\
\hline Marriage (Married) & 0.36 & $0.17-0.77$ & 0.008 & 0.30 & $0.09-0.93$ & 0.037 \\
\hline Work experience ( $\geq 3 \mathrm{yr}$ ) & 0.84 & $0.47-1.48$ & 0.544 & & & \\
\hline Drinking ( $>1$ day/wk) & 1.30 & $0.73-2.31$ & 0.381 & 1.33 & $0.66-2.67$ & 0.434 \\
\hline College graduate & 1.03 & $0.57-1.84$ & 0.929 & & & \\
\hline Poor sleep quality $(\mathrm{PSQI} \geq 6$ ) & 4.04 & $0.52-31.09$ & 0.180 & 3.51 & $0.42-29.59$ & 0.248 \\
\hline \multicolumn{7}{|l|}{ Psychosocial alarm } \\
\hline None & Reference & & & Reference & & \\
\hline Non-serious & 2.03 & $0.74-5.51$ & 0.167 & 2.15 & $0.59-7.88$ & 0.248 \\
\hline Serious & 5.67 & $2.00-16.04$ & 0.001 & 7.84 & $1.98-31.02$ & 0.003 \\
\hline
\end{tabular}

BMI, body mass index; PSQI, Pittsburgh Sleep Quality Index.

For the psychosocial alarm, the absence of psychosocial alarm is the reference group.

serious psychosocial alarm (aOR, 7.84; 95\% CI, 1.98-31.02; $P$ $=0.003)$ was the independent risk factor for $\mathrm{FD}$; however, marriage (aOR, $0.30 ; 95 \% \mathrm{CI}, 0.09-0.93 ; P=0.037$ ) was associated with a decreased risk of FD.

\section{Discussion}

Psychosocial factors, such as depression and anxiety, are important factors in the pathogenesis of FGIDs. ${ }^{13-17}$ Previously, it has been reported that nursing professionals who participate in a rotating shift schedule are at a higher risk of psychosocial problems than are those who work the day shift. ${ }^{18}$ However, the role of psychosocial risk in shift-working professionals in the development of FGIDs remains obscure. In the present study, psychosocial problems are more common in rotating shift workers than in day shift workers. In addition, psychological stress is an independent risk factor for IBS and FD. Therefore, we think that the high prevalence of psychological stress among nurses with rotating shift work may contribute to the development of FGIDs. Although our study did not show differences in the prevalence of 
FGID between the 2 groups, there was an increasing trend of FGIDs in subjects with rotating shift work. In addition, previous studies have demonstrated that rotating shift work increases the risk of FGIDs. ${ }^{7,8}$ Based on these results, we believe that there is an interactive effect between rotating shift work and psychosocial stress in the development of FGIDs.

Our data show that the prevalence of IBS is relatively low for nursing professionals compared to what previous studies have found. ${ }^{7,8}$ To exclude the possibility of short work experience being a confounding variable, we repeated the analyses excluding those subjects who had worked for less than 12 months on their current shift schedule. However, we observed a consistent finding that $17.5 \%$ of rotating shift workers reported the presence of IBS. It has been reported that the prevalence of IBS in Eastern countries is relative low compared with Western populations. ${ }^{19}$ In addition, the point prevalence of IBS identified using the ROME III criteria among healthy volunteers was $3.9 \%$ in Korea. ${ }^{20}$ Therefore, we believe that our data provide a precise estimation of the prevalence of IBS among nurse professionals who work rotating shift in Korea. However, this discrepancy may be due to differences in our study populations and setting due variations in educational status, income or workloads. Therefore, a multicenter study includes more subjects who work in various situations is needed.

Although neither PSQI scores nor the proportion of poor sleepers was an independent predictor of IBS and FGIDs in the regression analysis, our study showed very high PSQI score regardless of FD. A previous study reported that poor sleep quality occur frequently among hospital staff nurses. ${ }^{21}$ In this study, approximately $80 \%$ of hospital nurses had a total PSQI score of 5 or more. In addition, it has been reported that rotating shift nurses with FD in Korea showed similar PSQI score in nurses with FD. ${ }^{7}$ Based on these result, poor sleep quality is a common problem among nurses in Korea. In previous studies of nurses, poor sleep quality was an independent risk factor for FGIDs. ${ }^{7,8}$ Furthermore, a substantial proportion of hospital nurses had psychosocial stress in our study. Unfortunately, we did not investigate topics regarding medical help in nurses and nurse assistants for these problems. Therefore, further studies are required to investigate nurses' health issues and to develop a comprehensive strategy to cope with this health problem.

Three-quarters of the nursing personnel participating in this study reported the psychosocial alarm, and a quarter of them reported the serious psychosocial condition. This number is surprisingly high compared with that found in a previous study that sur- veyed general population using the same definition of psychosocial alarm, where the prevalence was $9 \%$ for the presence of alarm and $0.9 \%$ for the presence of serious alarm. ${ }^{12}$ However, our finding is consistent with previous findings that nursing personnel have increased risks of psychosocial morbidities. ${ }^{22}$ Occupational stressors such as patient care, team conflicts, role ambiguity and lack of autonomy have been suggested as some of the contributing factors. ${ }^{23}$ In addition, our study showed the increased psychosocial risk was associated with a rotating shift work schedule. Therefore, gastroenterologists should be aware of the association between work schedule, psychosocial factors and abnormal gastrointestinal symptoms when educating FGID patients.

Our study shows that marriage was associated with the decreased the risk of FD. This finding concurs with a previous study which reported the OR for the presence of FD in unmarried subjects was 1.4 compared with married individuals. ${ }^{24}$ Although a study reported the increased risk of FD with marriage, this was unexpected even to the authors as living with a partner is generally associated with increased quality of life. ${ }^{25}$ In addition, a study reported that married individuals had more emotional support that did single ones. ${ }^{26}$ This may explain how marriage decreased the risk of FD. In contrast, the association between marriage and IBS was not significant in our study. Although the effect of intimate relationships between IBS and marriage is well known, the statistical confirmation of their link is scarce in previous publications. ${ }^{27}$ Therefore, these results suggest that there may be different pathophysiologic mechanisms between IBS and FD.

Besides the common limitations of previous observational studies such as participation biases, unmeasured confounders and the problem of determining organic gastrointestinal disease without endoscopy, several limitations to this study are noteworthy. ${ }^{7,8}$ First, this study was limited in its single referral hospital setting, which may have led to selection bias. Second, there may have been unmeasured potential confounding factors. However, we considered various factor including psychosocial problems and proved the role of psychosocial factors on both IBS and FD after controlling confounders using multivariate regression analysis; therefore, we believe that our data provided additional information. Finally, we enrolled nursing professionals as subjects. However, nursing professionals are not representative of the general population, which may lead to selection bias. Therefore, further studies are needed to confirm our results among various groups of rotating shift workers.

In conclusion, our study demonstrated that psychosocial problems are more common among nursing workers in rotating 
shifts than those working the day shift. Furthermore, serious psychosocial alarm is an independent factor for the increased risk of both IBS and FD. Our results suggest that the high prevalence of psychosocial stress among nurses who work rotating shifts is associated with the development of FGIDs.

\section{References}

1. Drossman DA. The functional gastrointestinal disorders and the Rome III process. Gastroenterology 2006;130:1377-1390.

2. Lee YY, Chua AS. Investigating functional dyspepsia in Asia. J Neurogastroenterol Motil 2012;18:239-245.

3. Dapoigny M, Stockbrügger RW, Azpiroz F, et al. Role of alimentation in irritable bowel syndrome. Digestion 2003;67:225-233.

4. Locke GR 3rd, Weaver AL, Melton LJ 3rd, Talley NJ. Psychosocial factors are linked to functional gastrointestinal disorders: a population based nested case-control study. Am J Gastroenterol 2004;99: 350-357.

5. Smith RC, Greenbaum DS, Vancouver JB, et al. Psychosocial factors are associated with health care seeking rather than diagnosis in irritable bowel syndrome. Gastroenterology 1990;98:293-301.

6. Ibrahim NK, Battarjee WF, Almehmadi SA. Prevalence and predictors of irritable bowel syndrome among medical students and interns in King Abdulaziz University, Jeddah. Libyan J Med 2013;8: 21287.

7. Kim HI, Jung SA, Choi JY, et al. Impact of shiftwork on irritable bowel syndrome and functional dyspepsia. J Korean Med Sci 2013; 28:431-437.

8. Nojkov B, Rubenstein JH, Chey WD, Hoogerwerf WA. The impact of rotating shift work on the prevalence of irritable bowel syndrome in nurses. Am J Gastroenterol 2010;105:842-847.

9. Song KH, Jung HK, Min BH, et al. Development and validation of the Korean Rome III questionnaire for diagnosis of functional gastrointestinal disorders. J Neurogastroenterol Motil 2013;19:509-515.

10. Sohn SI, Kim do H, Lee MY, Cho YW. The reliability and validity of the Korean version of the Pittsburgh Sleep Quality Index. Sleep Breath 2012;16:803-812.

11. Levy RL, Olden KW, Naliboff BD, et al. Psychosocial aspects of the functional gastrointestinal disorders. Gastroenterology 2006;130:14471458 .

12. Lee YY, Waid A, Tan HJ, Chua AS, Whitehead WE. Rome III survey of irritable bowel syndrome among ethnic Malays. World J Gastroenterol 2012;18:6475-6480.

13. Blanchard EB, Scharff L, Schwarz SP, Suls JM, Balow DH. The role of anxiety and depression in the irritable bowel syndrome. Behav Res Ther 1990;28:401-405.

14. Jerndal P, Ringstrom G, Agerforz P, et al. Gastrointestinal-specific anxiety: an important factor for severity of GI symptoms and quality of life in IBS. Neurogastroenterol Motil 2010;22:646, e179.

15. Lackner JM, Quigley BM, Blanchard EB. Depression and abdominal pain in IBS patients: the mediating role of catastrophizing. Psychosom Med 2004;66:435-441.

16. Savas LS, White DL, Wieman M, et al. Irritable bowel syndrome and dyspepsia among women veterans: prevalence and association with psychological distress. Aliment Pharmacol Ther 2009;29:115-125.

17. Surdea-Blaga T, Băban A, Dumitrascu DL. Psychosocial determinants of irritable bowel syndrome. World J Gastroenterol 2012;18: 616-626.

18. Flo E, Pallesen S, Mageroy N, et al. Shift work disorder in nurses-assessment, prevalence and related health problems. PLoS One 2012;7: e33981.

19. Han SH, Lee OY, Bae SC, et al. Prevalence of irritable bowel syndrome in Korea: population-based survey using the Rome II criteria. J Gastroenterol Hepatol 2006;21:1687-1692.

20. Park H. Functional gastrointestinal disorders and overlap syndrome in Korea. J Gastroenterol Hepatol 2011;26(suppl 3):12-14.

21. Chien PL, Su HF, Heieh PC, Siao RY, Ling PY, Jou HJ. Sleep quality among female hospital staff nurses. Sleep Disord 2013;2013: 283490.

22. Wall TD, Bolden RI, Borrill CS, et al. Minor psychiatric disorder in NHS trust staff: occupational and gender differences. Br J Psychiatry 1997;171:519-523.

23. Eriksen W, Tambs K, Knardahl S. Work factors and psychological distress in nurses' aides: a prospective cohort study. BMC Public Health 2006;6:290.

24. Talley NJ, Zinsmeister AR, Schleck CD, Melton LJ 3rd. Smoking, alcohol, and analgesics in dyspepsia and among dyspepsia subgroups: lack of an association in a community. Gut 1994;35:619-624.

25. Moayyedi P, Forman D, Braunholtz D, et al. The proportion of upper gastrointestinal symptoms in the community associated with Helicobacter pylori, lifestyle factors, and nonsteroidal anti-inflammatory drugs. Leeds HELP Study Group. Am J Gastroenterol 2000; 95:1448-1455.

26. Cheng C, Hui WM, Lam SK. Psychosocial factors and perceived severity of functional dyspeptic symptoms: a psychosocial interactionist model. Psychosom Med 2004;66:85-91.

27. Gerson MJ, Gerson CD. The importance of relationships in patients with irritable bowel syndrome: a review. Gastroenterol Res Pract 2012;2012:157340. 